\title{
Sulfates as chromophores for multiwavelength photoacoustic imaging phantoms
}

Martina Fonseca

$\mathrm{Lu} \mathrm{An}$

Paul Beard

Ben Cox 


\title{
Sulfates as chromophores for multiwavelength photoacoustic imaging phantoms
}

\author{
Martina Fonseca, ${ }^{*}$ Lu An, Paul Beard, and Ben Cox \\ University College London, Department of Medical Physics and Biomedical Engineering, London, United Kingdom
}

\begin{abstract}
As multiwavelength photoacoustic imaging becomes increasingly widely used to obtain quantitative estimates, the need for validation studies conducted on well-characterized experimental phantoms becomes ever more pressing. One challenge that such studies face is the design of stable, well-characterized phantoms and absorbers with properties in a physiologically realistic range. This paper performs a full experimental characterization of aqueous solutions of copper and nickel sulfate, whose properties make them close to ideal as chromophores in multiwavelength photoacoustic imaging phantoms. Their absorption varies linearly with concentration, and they mix linearly. The concentrations needed to yield absorption values within the physiological range are below the saturation limit. The shape of their absorption spectra makes them useful analogs for oxyand deoxyhemoglobin. They display long-term photostability (no indication of bleaching) as well as resistance to transient effects (no saturable absorption phenomena), and are therefore suitable for exposure to typical pulsed photoacoustic light sources, even when exposed to the high number of pulses required in scanning photoacoustic imaging systems. In addition, solutions with tissue-realistic, predictable, and stable scattering can be prepared by mixing sulfates and Intralipid, as long as an appropriate emulsifier is used. Finally, the Grüneisen parameter of the sulfates was found to be larger than that of water and increased linearly with concentration. ( $\odot$ The Authors. Published by SPIE under a Creative Commons Attribution 3.0 Unported License. Distribution or reproduction of this work in whole or in part requires full attribution of the original publication, including its DOI. [DOI: 10.1117/1.JBO.22.12.125007]
\end{abstract}

Keywords: photoacoustic imaging; phantoms; chromophores; validation; thermoelastic efficiency; photostability.

Paper 170535R received Aug. 18, 2017; accepted for publication Nov. 20, 2017; published online Dec. 23, 2017.

\section{Introduction}

Photoacoustic imaging (PAI) is an emerging modality that can provide high-resolution three-dimensional (3-D) images of soft tissue. ${ }^{1-3}$ Since different endogenous, exogenous, and genetically encoded chromophores have different absorption spectra, photoacoustic images obtained at multiple wavelengths carry information on the distributions of these chromophores and, hence, information on tissue composition, physiology, and pathology. ${ }^{1,2,4,5}$ Nevertheless, the use of multiwavelength PAI for detecting, isolating, assessing the relative variation, or fully quantifying chromophore contributions in an accurate and robust manner is still challenging ${ }^{6,7}$ (a field broadly known as quantitative photoacoustic tomography). Part of the challenge is the access to suitable chromophores and phantoms for proof-of-concept, testing, validation, and optimization. Despite recent advances on the study of phantom matrix materials for general photoacoustic (PA) system characterization and quality control, ${ }^{8-12}$ for multiwavelength and quantitative PAI purposes further efforts are needed, with one of the crucial issues being finding suitable chromophores. ${ }^{13,14}$ It is essential for these to be well-characterized, well-behaved, and stable. They should also exhibit spectral variation and if possible be tissue-realistic, although the extent to which this is necessary will depend on the application. ${ }^{15,16}$

Overall, an ideal set of chromophores for phantom studies of multiwavelength and quantitative PAI will require some or all of the following characteristics, depending on the application:

*Address all correspondence to: Martina Fonseca, E-mail: martina.fonseca .13@ucl.ac.uk
1. Large photoacoustic generation efficiency (Grüneisen parameter) and low fluorescence quantum yield. This is necessary to ensure a good signal-to-noise ratio (SNR). ${ }^{17}$

2. Low propensity for peak power dependent transient effects such as ground-state bleaching/saturation ${ }^{13,18}$ (also referred to as resistance to transient photobleaching). This is necessary when using optical pulses with high peak power (pulsed photoacoustics), and when it is important that the specific absorption spectra are known accurately.

3. Low propensity for permanent photobleaching. ${ }^{9,13,17}$ This is essential when the imaging system requires the use of multiple illumination shots or when the same phantom will be used multiple times, both to prevent the absorption spectrum from changing over time and to maintain the photoacoustic signal strength.

4. Absorption spectra at visible or NIR wavelengths that are spectrally varying and unique and that display features similar to in vivo endogenous chromophores or exogenous contrast agents.

5. Compatibility with the phantom matrix in which they will be embedded or mixed, i.e., ability to form stable and homogeneous mixtures, and to measure (or calculate) the absorption spectrum in that mixture in a straightforward way. This is important when the absorber will be mixed directly with the matrix, rather 
than remaining separate within a tube, for instance. $^{10,13,19}$

6. The absorption coefficient $\mu_{\mathrm{a}}$ can be written as a linear sum of the specific absorption spectra of the constituent chromophores $\alpha_{i}$ weighted by their concentrations $c_{i}$,

$\mu_{\mathrm{a}}(\lambda)=\sum_{i=1}^{N} \alpha_{i}(\lambda) c_{i}$,

where $\lambda$ is the optical wavelength and $N$ is the total number of chromophores. This implies that

a. The specific absorption spectra $\alpha_{i}(\lambda)$ do not depend on the concentrations of the chromophores themselves, or, if they do, their dependence can be written using simple functional expressions. If this is not the case, then it would be necessary to measure and tabulate the spectra for all concentrations of interest, which could be very time-consuming. ${ }^{20}$

b. The specific absorption spectrum of one chromophore does not depend on the concentrations of the other chromophores present or any other constituents of the phantom. ${ }^{21}$

If these two conditions are satisfied, linear spectroscopic techniques can be used to estimate the concentrations from the absorption coefficients.

7. Fast, simple, repeatable, and cheap fabrication procedure that allows the chromophore to be produced in sufficient quantity.

The distinction can be usefully made between a surrogate, which is a chromophore whose photoacoustic properties are essentially the same as those of a given chromophore of interest, and an analog, which is a chromophore that has some properties, features, or behavior qualitatively similar to chromophores of interest, but which is not an exact photoacoustic surrogate. The former are useful for standardized assessment and quality control of commercial and/or clinical multiwavelength devices. The latter are crucial for validation, development, and optimization of multiwavelength and quantitative PA imaging techniques and systems in research and early-translation paradigms (the primary concern of this paper). In either case, finding chromophores that satisfy the various criteria listed is challenging.

Endogenous chromophores may be hard to isolate and have low temporal stability. ${ }^{22,23}$ Organic dyes can suffer from aggregation-related spectral shifts and often display transient and permanent photobleaching, e.g., cyanine-based dyes, ${ }^{9,13,24}$ azobased $^{5}$ dyes, and basic dyes such as cresyl violet. ${ }^{24}$ Gold nanoparticles can also exhibit changes in absorption after prolonged exposure, ${ }^{9}$ with the added difficulty that their photoacoustic efficiency is not straightforward to model and characterize. ${ }^{25,26}$ Inorganic absorbers such as Prussian blue and chloride salts such as $\mathrm{CuCl}_{2} \cdot 2 \mathrm{H}_{2} \mathrm{O}$ and $\mathrm{NiCl}_{2} \cdot 6 \mathrm{H}_{2} \mathrm{O}$ are highly photostable and have suitable spectra. ${ }^{13}$ However, we have observed that the specific absorption spectra of the two chloride salts are affected both by their own concentrations and also by the presence of each other when dissolved in a mixture. Where other matrices besides water are concerned, another issue that has to be factored in is that chromophores often have their specific absorption spectra affected when mixed with those matrices, e.g., there have been reports of Prussian blue absorption behaving unpredictably when mixed with Intralipid ${ }^{13}$ of redshifting of the spectrum of azo-dyes Direct Red 81 and Evans Blue in gelatin; ${ }^{10}$ of blueshifting and amplitude rise of $\mathrm{CuCl}_{2} \cdot 2 \mathrm{H}_{2} \mathrm{O}$ in gelatin; ${ }^{13,21}$ and of considerable changes in cyanine dye ICG when put in different solvents. ${ }^{20}$

The use of aqueous solutions of sulfate salts such as copper sulfate and nickel sulfate in some PA studies seems to suggest that these inorganic compounds may be suitable as chromophores for PAI validation studies. Su et al., ${ }^{27}$ Brecht et al., ${ }^{28}$ and Wang et al. ${ }^{29}$ used tube phantoms filled with aqueous solutions of sulfate salts, submersed in a bath of intralipid, to help study various aspects of the performance of a wholebody small animal PAI system. More recently, Petrova et al. ${ }^{30}$ gave a proof-of-principle demonstration of a photoacoustic method for temperature monitoring using aqueous solutions of both copper and nickel sulfate in tube phantoms.

These studies suggest that sulfates could be suitable chromophores for well-controlled phantom studies, but a full characterization and evaluation has not been reported. In this paper, we extend the previous work by providing detailed experimental measurements of the optical and PA properties of aqueous solutions and emulsions of nickel sulfate hexahydrate and copper sulfate pentahydrate-namely, their absorption spectra, absorption linearity, absorption behavior in mixtures of sulfate species, transient and permanent photostability, absolute Grüneisen characteristics, and compatibility with intralipid as a scattering medium-and present the case for them as near-ideal chromophores for use in multiwavelength PAI phantoms.

\section{Tabulated Properties}

The molecular weight and solubility of sulfates have been measured previously and are well-known; they are given here in Table $1 .{ }^{31}$ The solubility is an important metric since it reveals the maximum absorption that can be reached before linearity of absorption with concentration is compromised. Information on $\mathrm{pH}$ and density of $\mathrm{NiSO}_{4} \cdot 6 \mathrm{H}_{2} \mathrm{O}$ has also been documented. ${ }^{32}$

The experiments were performed on the compounds in their hydrated form, but for ease of notation they will often be referred to simply as copper sulfate, $\mathrm{CuSO}_{4}$, and nickel sulfate, $\mathrm{NiSO}_{4}$.

\section{Optical Absorption}

The absorption spectra, absorption linearity with concentration, and absorption linearity of mixtures were measured for solutions of copper and nickel sulfate using a dual-beam spectrophotometer (Lambda 750S, Perkin Elmer, Waltham, Massachusetts), with water acting as the blank. The mother batch solutions for each species were chosen to have molar concentrations close to

Table 1 Literature-reported properties of copper and nickel sulfate. ${ }^{31}$

\begin{tabular}{lccc} 
Compound & $\begin{array}{c}\text { Molecular } \\
\text { formula }\end{array}$ & $\begin{array}{c}\text { Molecular } \\
\text { weight }(\mathrm{g} / \mathrm{mol})\end{array}$ & Solubility at $20^{\circ} \mathrm{C}$ \\
\hline $\begin{array}{l}\text { Copper sulfate } \\
\text { pentahydrate }\end{array}$ & $\mathrm{CuSO}_{4} \cdot 5 \mathrm{H}_{2} \mathrm{O}$ & 249.68 & $320 \mathrm{~g} / \mathrm{l} \simeq 1.28 \mathrm{M}$ \\
$\begin{array}{l}\text { Nickel sulfate } \\
\text { hexahydrate }\end{array}$ & $\mathrm{NiSO}_{4} \cdot 6 \mathrm{H}_{2} \mathrm{O}$ & 262.85 & $625 \mathrm{~g} / \mathrm{l} \simeq 2.37 \mathrm{M}$ \\
\hline
\end{tabular}




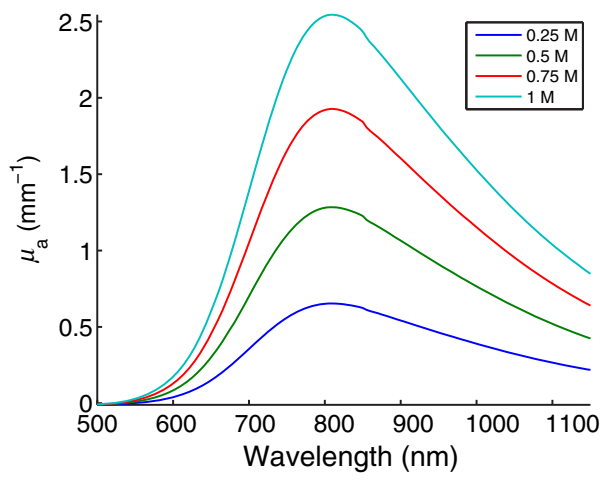

(a) $\mathrm{CuSO}_{4}$

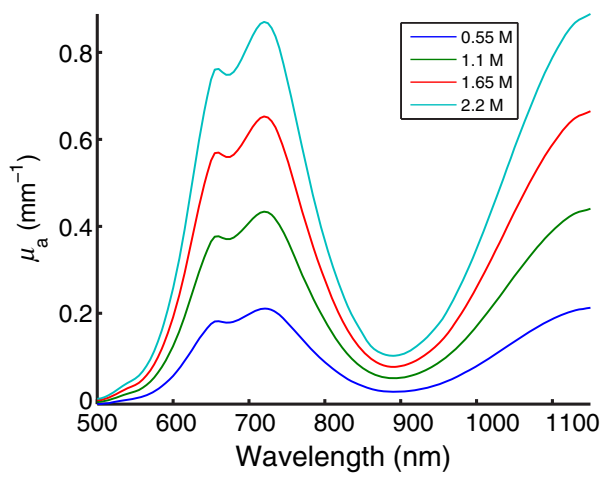

(c) $\mathrm{NiSO}_{4}$

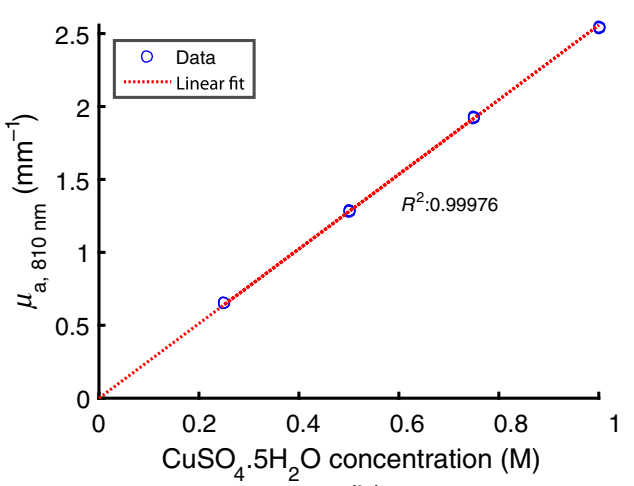

(b)

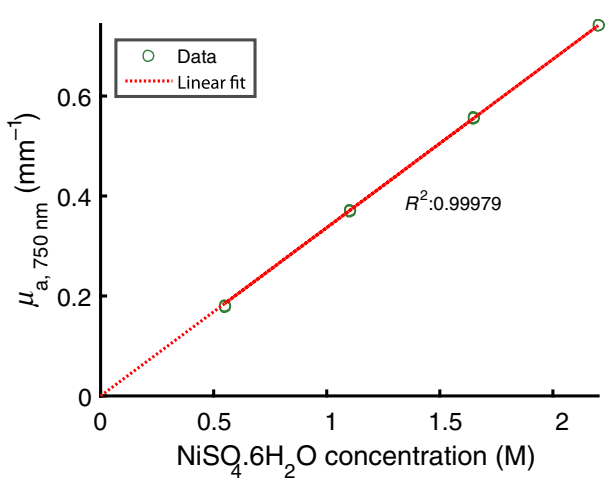

(d)

Fig. 1 Optical absorption of solutions of either $\mathrm{NiSO}_{4}$ and $\mathrm{CuSO}_{4}$ at various levels of dilution. (a, c) Absorption spectra of four levels of dilution (error bars omitted from the figures as they could not be properly resolved - the standard deviation of three measurements per solution was below $1 \%$ of the mean for most cases and wavelengths); (b, d) plotting optical absorption versus concentration at a prominent wavelength to highlight that absorption linearity with concentration is respected. Three measurements are shown per concentration, but their overlap is such that they cannot be easily resolved when plotted.

the solubility limit, $c_{\mathrm{NiSO}_{4}, \mathrm{~b}}=2.2 \mathrm{M}, c_{\mathrm{CuSO}_{4}, \mathrm{~b}}=1 \mathrm{M}$, where subscript $\mathrm{b}$ stands for batch. They were then diluted to obtain solutions with $c_{\mathrm{NiSO}_{4}}=\{0.55,1.1,1.65,2.2\} \mathrm{M}$ and $c_{\mathrm{CuSO}_{4}}=$ $\{0.25,0.5,0.75,1\} \mathrm{M}$, respectively. Mixtures of the mother solutions of $\mathrm{NiSO}_{4}$ and $\mathrm{CuSO}_{4}$ were also prepared according to the following ratios of $c_{\mathrm{NiSO}_{4}, \mathrm{~b}}$ to $c_{\mathrm{CuSO}_{4}, \mathrm{~b}}: 1: 3,2: 2$, and $3: 1$. For each solution, three measurements were performed by emptying the cuvette, extracting a new portion of the solution, and remeasuring.

\subsection{Absorption Linearity with Concentration}

Figure 1 shows the measured absorption coefficient spectra of the various solutions of $\mathrm{NiSO}_{4}$ and $\mathrm{CuSO}_{4}$, at different levels of dilution. Absorption increases linearly with concentration. This linearity [criterion 6(a)] was respected across the studied range-namely, at 600, 700, 800, 900, 1000, and $1100 \mathrm{~nm}$; the coefficients of correlation obtained were $0.9965,0.9997$, 0.9994, 0.9886, 0.9978, and 0.9994, respectively, for $\mathrm{NiSO}_{4}$ and $0.9972,0.9998,0.9998,0.9999,0.9998,0.9998$, respectively, for $\mathrm{CuSO}_{4}$. This is also shown in Figs. 1(b) and 1(d) through the linear fits near peak wavelengths $(750 \mathrm{~nm}$ for $\mathrm{NiSO}_{4}$ and $810 \mathrm{~nm}$ for $\mathrm{CuSO}_{4}$ ).

The absorption of mixtures made from the mother batch solutions was also measured. Figure 2 shows the measured spectra of these mixtures (labeled meas for measured) in comparison with those theoretically expected by considering the measured

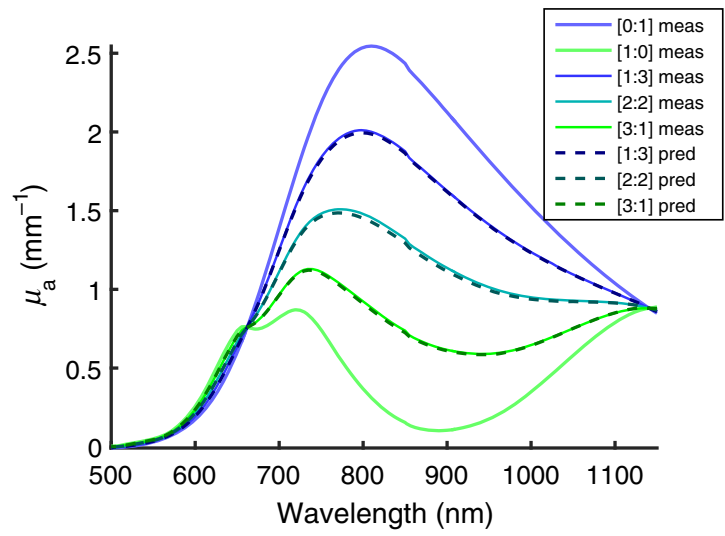

Fig. 2 Absorption spectra of mother batch solutions of $\mathrm{NiSO}_{4}$ and $\mathrm{CuSO}_{4}$ and of three mixtures of these, at ratios 1:3, 2:2, and 3:1. The mixtures have a measured absorption spectrum (meas) that matches the predicted spectrum (pred). The predictions are made through simple linear combination of the measured spectra of the mother batch solutions of nickel sulfate, $c_{\mathrm{NiSO}_{4}, \mathrm{~b}}([1: 0])$, and copper sulfate, $c_{\mathrm{CuSO}_{4}, \mathrm{~b}}([0: 1])$, weighted by the expected ratios.

mother batch spectra and applying Eq. (1) (labeled pred for predicted). It can be seen that the measured spectra for the mixtures accurately reflect the linear sum of the unmixed absorptions of the two individual chromophores-criterion 6 (b) is respected. 


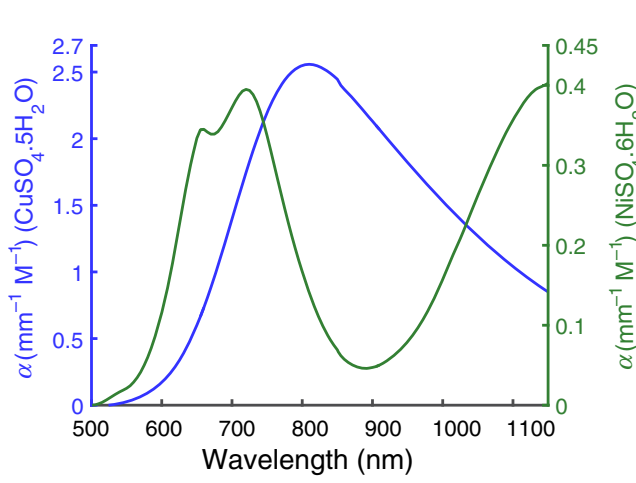

(a)
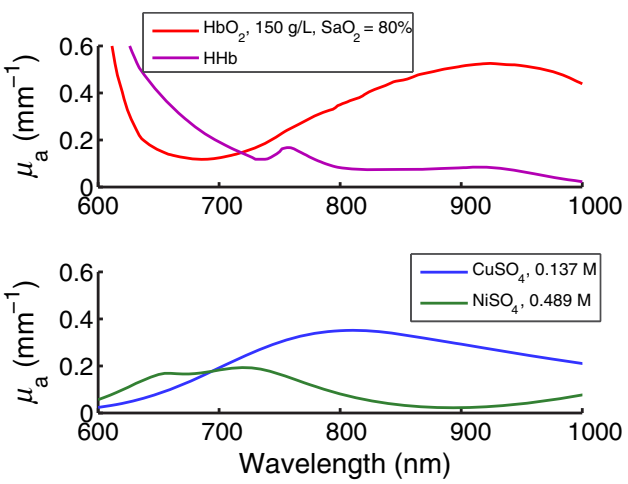

(b)

Fig. 3 Absorption spectra of sulfate salts and of oxy- and deoxyhemoglobin. (a) Specific absorption spectra of $\mathrm{NiSO}_{4}$ and $\mathrm{CuSO}_{4}$; (b) $\mathrm{NiSO}_{4}$ and $\mathrm{CuSO}_{4}$ as analogs for deoxy- and oxyhemoglobin. Spectra for deoxy- and oxyhemoglobin are shown as their partial/individual contributions to absorption of total blood with $150 \mathrm{~g} / \mathrm{L}$ hemoglobin and $80 \%$ saturation. Spectra for $\mathrm{NiSO}_{4}$ and $\mathrm{CuSO}_{4}$ are plotted for concentrations where absorption would match that of deoxy- and oxyhemoglobin, respectively, at $800 \mathrm{~nm}$.

\subsection{Absorption Spectra}

Figure 3(a) shows the specific absorption spectrum of each compound, $\alpha(\lambda)$. The spectrum of each of the two compounds is rich in features and the maximum achievable absorption is in the range of interest for biological tissue and contrast agents. Furthermore, when considered together, the two chromophores are also clearly unique, with different features and gradients. These spectral attributes make the sulfates suitable as analogs for a variety of multiwavelength-based studies focused on both quantification-related systems and methods.

As for the subsidiary interest of having access to surrogates, although the sulfates cannot be straightforwardly considered to perfectly mirror select endogenous or exogenous chromophores in typical (pre-)clinical scenarios, it is of interest to note some parallelisms found among $\mathrm{CuSO}_{4}, \mathrm{NiSO}_{4}$, and oxy- and deoxyhemoglobin, respectively. We consider that these can be exploited in, e.g., studies focused on ratiometric concentration estimation. As a case study, Fig. 3(b) plots the absorption spectra of oxy- and deoxyhemoglobin in a typical physiological blood sample-with total hemoglobin concentration $c_{\mathrm{HbT}}=$ $150 \mathrm{~g} / \mathrm{L}$ and oxygen saturation $\mathrm{SO}_{2}=80 \%,{ }^{22}$ as well as the spectra of a $0.137-\mathrm{M} \mathrm{CuSO}_{4}$ solution and $0.489-\mathrm{M} \mathrm{NiSO}_{4}$ solution, where these molar concentrations were chosen to match the absorption of oxy- and deoxyhemoglobin at $800 \mathrm{~nm}$, respectively. Though it is clear that the spectra differ (namely, in peak position and in monotony near $600 \mathrm{~nm}$ ), similar characteristics can be seen for both pairs in the typical near-infrared window (650 nm onward) - the pairs of spectra cross-over once and near $700 \mathrm{~nm}$ in both cases; also, both $\mathrm{CuSO}_{4}$ and oxyhemoglobin $\left(\mathrm{HbO}_{2}\right)$ have similar spectral shapes, with a very broad main peak, though one is centered at $815 \mathrm{~nm}$ and the other closer to $950 \mathrm{~nm}$; finally, deoxyhemoglobin $\left(\mathrm{HHb}_{2}\right)$ has a mainly decreasing or plateauing behavior that is fairly well mirrored by $\mathrm{NiSO}_{4}$. As a further case study, if emulating the isosbestic point of hemoglobin near $800 \mathrm{~nm}$ is desired (i.e., the wavelength where, for a certain hemoglobin concentration $c_{\mathrm{HbT}}$, the total absorption will remain unchanged regardless of the level of oxygen saturation, $\mathrm{SO}_{2}$ ), mixtures can be made from mother batch solutions of $\mathrm{NiSO}_{4}$ and $\mathrm{CuSO}_{4}$ that have a relative molar ratio of $\sim 14.28$ to 1 , i.e., $c_{\mathrm{NiSO}_{4}, \mathrm{~b} 2}[M]=14.28 c_{\mathrm{CuSO}_{4}, \mathrm{~b} 2}[M]$.

\subsection{Absorption at High Peak Power}

The aim in this section is to verify that the compounds do not exhibit transient or permanent photobleaching when illuminated with high peak power pulses.

To verify resistance to transient photobleaching (criterion 2), the absorption spectra obtained with the spectrophotometerwhich employs low power CW illumination-were compared with spectra that were measured photoacoustically using a ns-pulsed high peak power source. A fiber-coupled Nd:YAG pumped wavelength-tunable optical parametric oscillator system was used (SpitLight 600, Innolas, Krailling, Germany), providing 6-ns pulses at a $30-\mathrm{Hz}$ pulse repetition rate, with an incident energy of $\sim 7.3 \mathrm{~mJ}$ at $750 \mathrm{~nm}$ and $\sim 6.2 \mathrm{~mJ}$ at $810 \mathrm{~nm}$. The photoacoustic spectroscopy (PAS) signals were detected using a $10 \mathrm{~mm} \times 10 \mathrm{~mm} \times 50 \mu \mathrm{m}$ piezoelectric polyvinylidene fluoride film bonded to a PMMA backing ${ }^{24}$ and the absorption coefficient was retrieved by curve-fitting to the exponential decay expected of a photoacoustic signal generated in a homogeneous medium, $S(t)=\kappa \Gamma \Phi_{0} \mu_{\mathrm{a}} e^{-\mu_{\mathrm{a}} c t}(\mathrm{~V})$, where $t$ is time, $\kappa$ is a scaling parameter, $\Phi_{0}$ is the incident energy, $\Gamma$ is the Grüneisen parameter, and $c$ is the sound speed. ${ }^{13,24}$ Figure 4(a) shows the good agreement of the spectrophotometer ([S]) and PAS ([P]) absorption spectra, both in absolute units $\left(\mathrm{mm}^{-1}\right)$. This agreement in $\mu_{\mathrm{a}}$ for a wide spectral range supports the hypothesis that no transient photobleaching is occurring (transient phenomena at high peak power would manifest themselves in PAS through lowered absorption and/or altered spectral characteristics ${ }^{13,17}$ ) - criterion 2 is respected. For the photoacoustically derived data, only the wavelengths that yielded a goodness of fit of $R^{2}>0.98$ for the exponential are displayed - the instances of poor and unreliable exponential fitting were due to the absorption of $\mathrm{NiSO}_{4}$ being too low at some wavelengths, generating poor quality photoacoustic signals. The results go in line with a previous study by BalderasLopez and Díaz-Reyes, ${ }^{33}$ who reported similar optical absorption estimates for copper sulfate at a wavelength of $658 \mathrm{~nm}$ between a spectrometer-based method and a PA method.

The same PAS system was used to verify photostability under long-term exposure to high peak power pulses, with newly prepared samples of $\mathrm{NiSO}_{4}$ and $\mathrm{CuSO}_{4}$ being continuously excited 


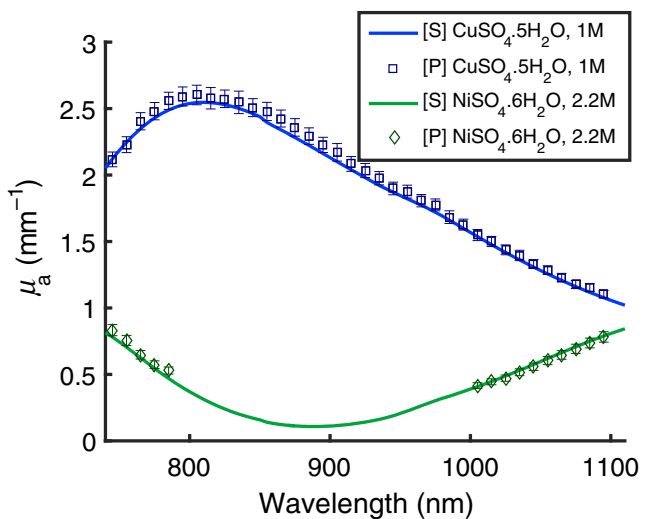

(a)

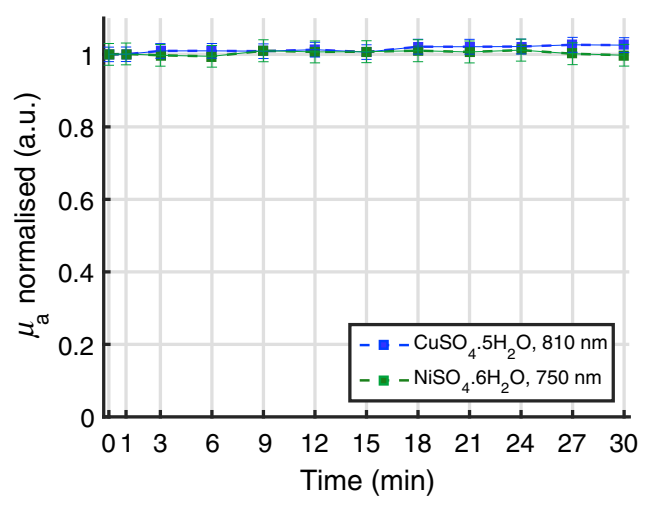

(b)

Fig. 4 Photostability assessment. (a) Spectroscopically (solid line) and photoacoustically (data points) derived absorption spectra, showing agreement. Error bars reflect intersample variation and uncertainty of the PAS system methodology; (b) photostability during 30 min irradiation (54,000 pulses). Error bars reflect uncertainty of the PAS system methodology.

for $30 \mathrm{~min}(54,000$ pulses total at $30 \mathrm{~Hz})$. Their photoacoustic signals were recorded every $3 \mathrm{~min}$, from which the change in fitted $\mu_{\mathrm{a}}$ over time could be studied. ${ }^{17,24}$ The wavelengths chosen were close to the absorption maxima of each compound. Figure 4(b) shows that no long-term photobleaching was observed since the photoacoustically measured absorption coefficients were constant over the exposure time period (criterion 3 is respected).

\section{Optical Scattering}

Aqueous solutions of both $\mathrm{CuSO}_{4}$ and $\mathrm{NiSO}_{4}$ are nonscattering. A lipid emulsion, such as Intralipid, is commonly used to confer tissue-like scattering properties to solutions and phantom media. ${ }^{16}$ However, mixing sulfate (or chloride) salt solutions with Intralipid is not stable, ${ }^{34,35}$ which is problematic for PA studies since this means that the optical properties will be inhomogeneous, unpredictable, and dynamic over time. Salts are known to disrupt hydrophobic/hydrophilic interactions between emulsion molecules, ${ }^{36}$ which at the macroscopic level is manifested by emulsions typically separating into two phases and becoming cloudy. To better assess this, Intralipid emulsions with added concentrations of nickel and copper sulfate, providing an absorption comparable to typical background biological tissue, were prepared. Figure 5 shows the 12 -h stability of $1 \% \mathrm{w} / \mathrm{v}$ Intralipid mixtures with different additives: while Intralipidonly (v) and Intralipid + India ink (i) mixtures were stable, those with added sulfates (ii-iv) were unstable, creating two layers: a layer rich in Intralipid on top of an aqueous translucent layer. The macroscopic separation occurred less than half an hour after mixing.

It was hypothesised that adding an emulsifier such as Tween20 (polysorbate 20) to sulfate aqueous solutions before mixing with Intralipid could stabilize Intralipid emulsions. ${ }^{37,38}$ To test this, $1 \% \mathrm{w} / \mathrm{v}$ Intralipid solutions with $0.033 \mathrm{M} \mathrm{NiSO}_{4}$ were prepared, with concentrations of Tween $c_{\text {tween }}=\{0,0.05,0.5,5\} \%$ $\mathrm{v} / \mathrm{v}$. A control $1 \% \mathrm{w} / \mathrm{v}$ Intralipid solution was also prepared,

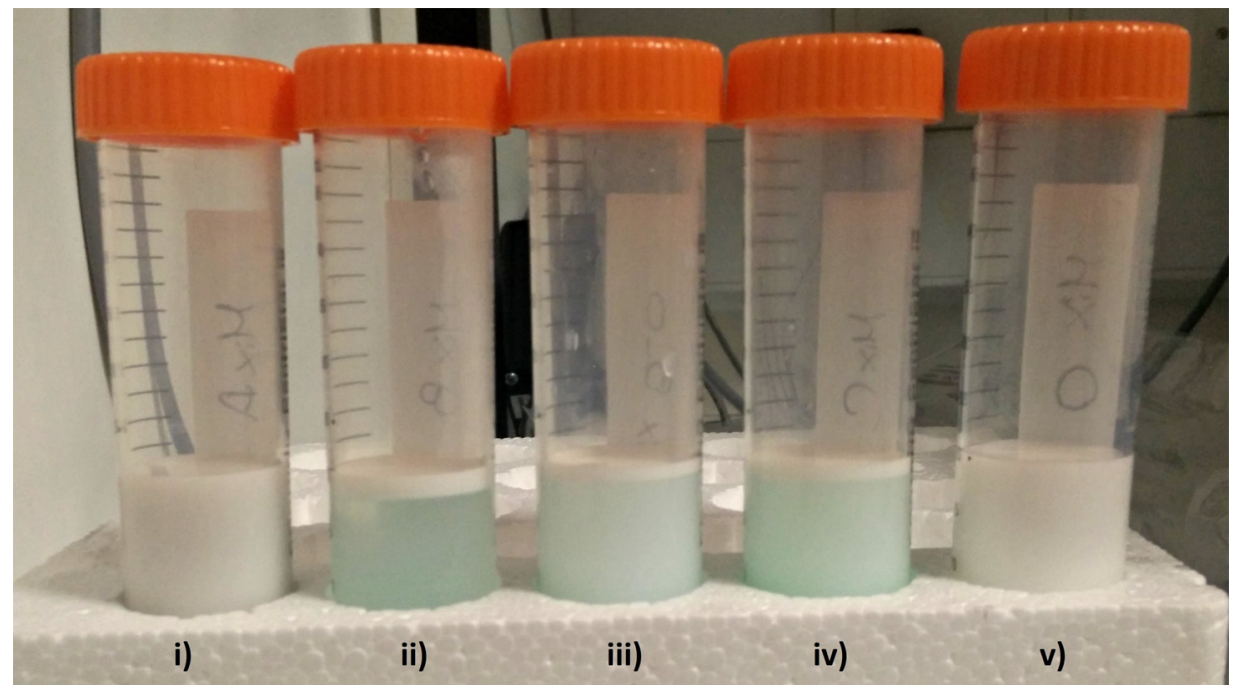

Fig. $512 \mathrm{~h}$ stability of mixtures. All solutions are made of $1 \% \mathrm{w} / \mathrm{v}$ intralipid in water. From left to right, they have different absorbers: (i) added India ink, yielding $\mu_{\mathrm{a}} \simeq 0.008 \mathrm{~mm}^{-1}$ at $750 \mathrm{~nm}$; (ii, iii) added nickel and copper sulfate, yielding $c_{\mathrm{NiSO}_{4}}=0.011 \mathrm{M}^{-1}, c_{\mathrm{CuSO}_{4}}=0.005 \mathrm{M}^{-1}$; (iv) added nickel sulfate, yielding $c_{\mathrm{NiSO}_{4}}=0.033 \mathrm{M}^{-1} ;(\mathrm{v})$ no additional absorber. 
Table 2 Samples for reduced scattering measurements.

\begin{tabular}{lcccc}
\hline Samples & $\begin{array}{c}\text { Intralipid } \\
(\% \mathrm{w} / \mathrm{v})\end{array}$ & $\mathrm{NiSO}_{4}(\mathrm{M})$ & $\mathrm{CuSO}_{4}(\mathrm{M})$ & $\begin{array}{c}\text { Tween-20 } \\
(\% \mathrm{v} / \mathrm{v})\end{array}$ \\
\hline $3 . \mathrm{a}$ & 1 & 0 & 0 & 0 \\
$3 . \mathrm{b}$ & 1 & 0 & 0 & 0.025 \\
3.c & 1 & 0.0165 & 0 & 0 \\
$3 . \mathrm{d}$ & 1 & 0.0165 & 0 & 0.025 \\
$3 . \mathrm{e}$ & 1 & 0.0495 & 0 & 0 \\
$3 . \mathrm{f}$ & 1 & 0.0495 & 0 & 0.075 \\
$3 . \mathrm{g}$ & 1 & 0 & 0.0037 & 0 \\
$3 . \mathrm{h}$ & 1 & 0 & 0.0037 & 0.025 \\
\hline
\end{tabular}

with no added sulfates or Tween. The control solution and all solutions containing Tween presented a single homogeneous phase for up to $48 \mathrm{~h}$ after sample preparation and homogenization, while the sample with added sulfate but no emulsifier started separating into two layers less than half an hour after preparation. This gave a visual indication that Tween-20, even at a concentration as low as $0.05 \% \mathrm{v} / \mathrm{v}$, could be used to stabilize mixtures with this level of salt concentration.

To assess this more rigorously, the reduced scattering coefficient $\mu_{\mathrm{s}}^{\prime}$ was measured for a new batch of solutions (Table 2), both immediately after preparation and $48 \mathrm{~h}$ later. To obtain the reduced scattering coefficients, total transmittance, and total reflectance measurements were made using a 100-mm integrating sphere mounted in the dual-beam spectrophotometer. Measurements were made between 500 and $1200 \mathrm{~nm}$, with three repeats per solution. The inverse-adding-doubling algorithm ${ }^{39}$ was then used to calculate $\mu_{\mathrm{s}}^{\prime}$. The scattering anisotropy factor was set to $g=0.6$. $^{40}$

Figure 6(a) shows that the addition of sulfates-both $\mathrm{NiSO}_{4}$ and $\mathrm{CuSO}_{4}$ - to Intralipid significantly affects the scattering behavior and that this behavior varies nonlinearly with sulfate concentration. The samples were homogenized before measuring, so the change in scattering coefficient of the emulsions containing sulfates is not due to macroscopic phase separation but due to changes in the emulsion composition itself at the molecular/assembly level. In all cases where Tween was present, the reduced scattering coefficient was similar to the values obtained in the Intralipid-only sample and remained stable and well-matched even after $48 \mathrm{~h}$. The effect of the sulfates and Tween can also be observed clearly through the total transmittance curves in Fig. 6(b). Theoretically, the addition of $0.0165 \mathrm{M}$ of $\mathrm{NiSO}_{4}$ should increase absorption marginally and, therefore, reduce total transmittance marginally. Nevertheless, measurements show a considerable increase in total transmittance for sample 3.c. The equivalent solution with presence of $0.025 \%$ $\mathrm{v} / \mathrm{v}$ of Tween (sample 3.d) successfully avoided this effect, yielding a total transmittance curve identical to the one obtained with the Intralipid-only sample (similar behavior was also seen with samples 3.e and 3.g and their respective Tween-added counterparts - data not shown to maintain visual clarity). All of the previous show that criterion 5 can be respected when Intralipid is the desired matrix material.

\section{Grüneisen Parameter}

The Grüneisen parameter, $\Gamma$, of the sulfate solutions was found to depend on their concentration. To investigate this dependence, solutions at five concentrations were prepared for each compound: $c_{\mathrm{NiSO}_{4}}=\{0.275,0.55,1.1,1.65,2.2\} M$ and $c_{\mathrm{CuSO}_{4}}=\{0.125,0.25,0.5,0.75,1\} M$. Grüneisen parameter estimates were obtained with the PAS setup described in Sec. 3.3, with water used as a reference. ${ }^{24}$ Measurements of peak-to-peak photoacoustic amplitude spectra were recorded for water $\left[S_{0 \text {,water }}(\lambda)\right]$ and each sample $\left[S_{0, \text { sample }}(\lambda)\right]$ at wavelengths where the water absorption has a prominent peak and is therefore dominant -1400 to $1500 \mathrm{~nm}$. The ratio $\Gamma_{\text {sample }} / \Gamma_{\text {water }}$ is given by

$\left[\frac{S_{0, \text { sample }}(\lambda)}{\mu_{\text {a,sample }}(\lambda)}\right]=\frac{\Gamma_{\text {sample }}}{\Gamma_{\text {water }}}\left[\frac{S_{0, \text { water }}(\lambda)}{\mu_{\text {a,water }}(\lambda)}\right]$.

It is therefore possible, using a linear least squares fit to the measurements at different wavelengths, to retrieve $\Gamma_{\text {sample }} /$ $\Gamma_{\text {water }} \cdot \mu_{\mathrm{a} \text {,water }}(\lambda)$ and $\mu_{\mathrm{a} \text {,sample }}(\lambda)$ were measured in the spectrophotometer.

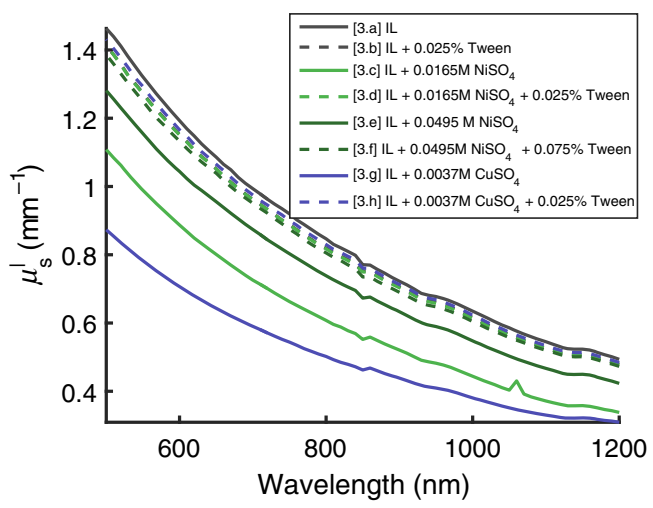

(a)

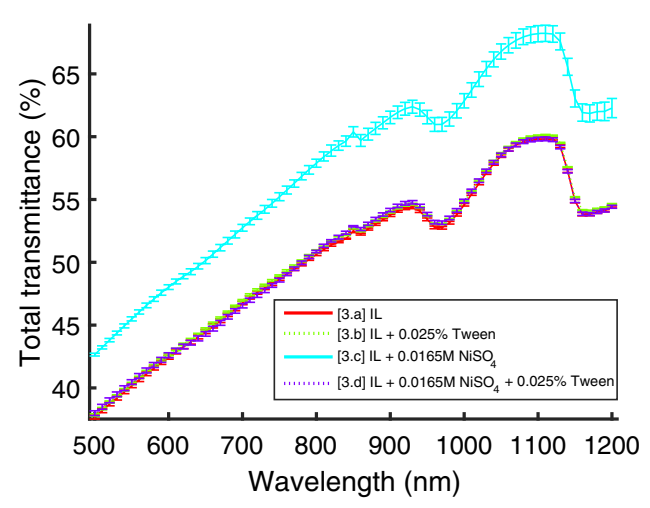

(b)

Fig. 6 (a) Reduced scattering coefficient of Intralipid 1\% w/v (IL) emulsions with and without addition of Tween and/or sulfates. All emulsions have similar behavior, except those with added sulfates and no Tween. (b) Raw total transmittance measurements for a selection of emulsions. The emulsion with added $\mathrm{NiSO}_{4}$ but no Tween shows a clearly disparate behavior. 

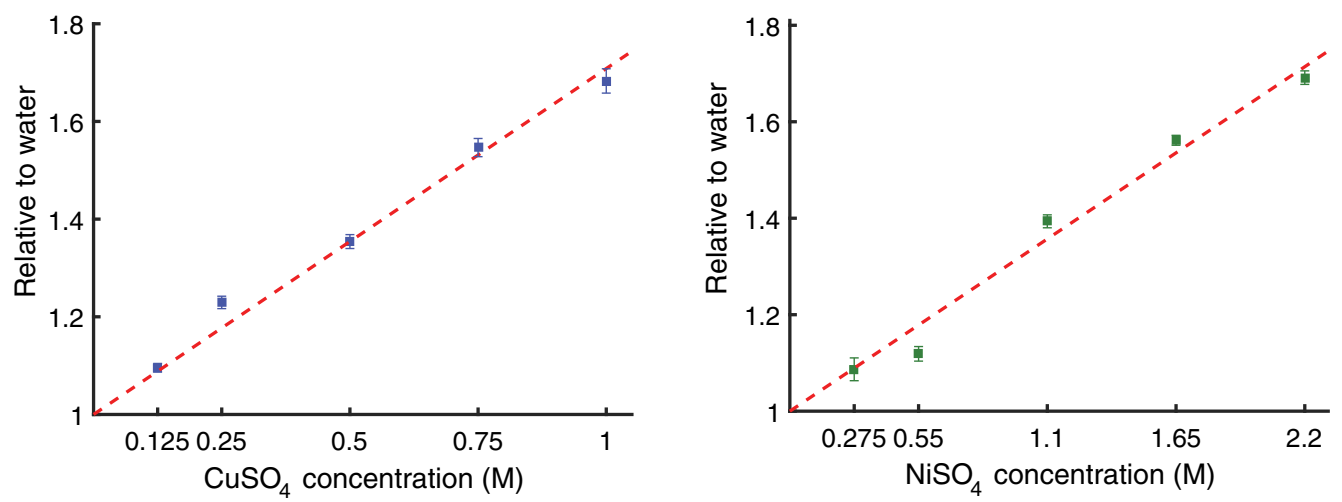

Fig. 7 Grüneisen parameter dependence with concentration, measured relative to water. Linearity is observed as shown by the linear fit (dashed line).

Table 3 Calculated factors accounting for concentration-dependency of the Grüneisen parameter of copper and nickel sulfate.

\begin{tabular}{lccc} 
Compound & $\beta$ factor $\left(M^{-1}\right)$ & $\begin{array}{c}\beta \text { factor standard } \\
\text { uncertainty }\end{array}$ & $\begin{array}{c}\text { Temperature in } \\
\text { solution }\left({ }^{\circ} \mathrm{C}\right)\end{array}$ \\
\hline $\mathrm{CuSO}_{4} .5 \mathrm{H}_{2} \mathrm{O}$ & 0.708 & \pm 0.025 & $23.0 \pm 0.2$ \\
$\mathrm{NiSO}_{4} \cdot 6 \mathrm{H}_{2} \mathrm{O}$ & 0.325 & \pm 0.015 & $22.6 \pm 0.2$ \\
\hline
\end{tabular}

By making measurements at different levels of dilution, the Grüneisen parameter was found to increase linearly with concentration (Fig. 7), where the slopes $\beta_{\mathrm{NiSO}_{4}}$ and $\beta_{\mathrm{CuSO}_{4}}$ were found through linear regression and are given in Table 3. The linear behavior of $\Gamma$ with salt concentration is consistent with previous observations: Petrova et al. ${ }^{30,41}$ characterized the relative change of Grüneisen parameter with temperature for both sulfates and found that the temperature point of null PA signal (null Grüneisen parameter) changed linearly with salt concentration. Furthermore, Grüneisen behavior in chloride salts had also been previously found to vary linearly with concentration. ${ }^{13}$ It is possible that an increase in sound speed is coupled to this behavior (although this has not been shown), as they are linked thermodynamically. However, the expected variation is within the range of variability in soft tissue. ${ }^{42,43}$ For an aqueous solution of $\mathrm{CuSO}_{4}$ and/or $\mathrm{NiSO}_{4}$, the Grüneisen parameter could be approximated by the following empirical formula:

$\Gamma_{\text {solution }}=\Gamma_{\text {water }}\left(1+\beta_{\mathrm{NiSO}_{4}} c_{\mathrm{NiSO}_{4}}+\beta_{\mathrm{CuSO}_{4}} c_{\mathrm{CuSO}_{4}}\right)$

as has previously been assumed for chloride aqueous compounds. ${ }^{13}$ This empirical joint behavior was also further tested with measurements on a mixture.

The Grüneisen levels provided by these solutions are higher than water, which brings increased SNR, a useful aspect for many studies (criterion 1 is respected). Furthermore, the levels can be considered tissue-realistic. ${ }^{23}$ As an example of similarity, if we consider the hypothetical sulfate mixture shown in Fig. 3(b) (bottom), which emulates absorption at $800 \mathrm{~nm}$ for oxy- and deoxyhemoglobin in blood with $150 \mathrm{~g} / \mathrm{L}$ hematocrit at $80 \%$ saturation, this mixture would be expected to have a $\Gamma_{\text {solution }}$ of 0.206 based on the empirical formula, which falls within the range reported for blood, 0.152 to $0.226 .{ }^{44}$ Interestingly, the linear dependence with concentration itself

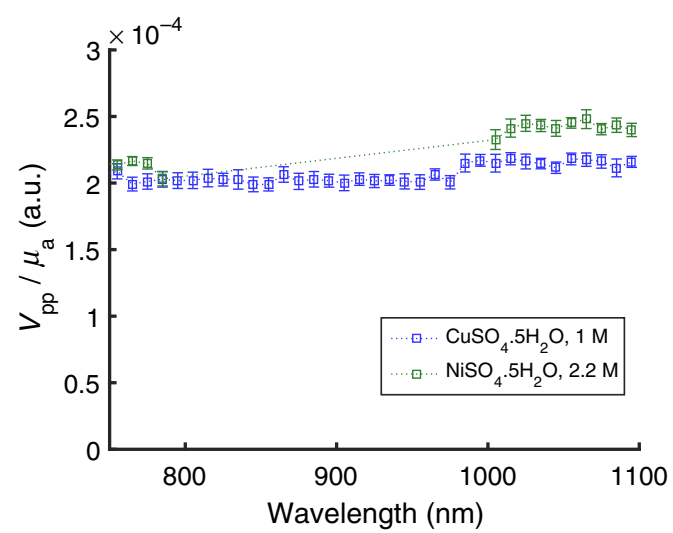

Fig. 8 Ratio between peak-to-peak voltage reading $V_{\mathrm{pp}}$ and exponentially fitted $\mu_{\mathrm{a}}$.

may also be a physiologically relevant characteristic: it has been shown that the Grüneisen parameter in red blood cell suspensions also displays a linear type of behavior with hemoglobin concentration, ${ }^{23} \Gamma_{\mathrm{RBCs}}=0.124+0.04995 f_{150 \mathrm{~g} / \mathrm{L}}$ at $22^{\circ} \mathrm{C}$, where $f_{150 \mathrm{~g} / \mathrm{L}}$ is the concentration of hemoglobin given as a fraction of $150 \mathrm{~g} / \mathrm{L}$, a typical physiological hematocrit. If we rewrite the expression as a function of $\Gamma_{\text {water }}=0.124$, where 0.124 is a realistic literature value for water in the $22^{\circ} \mathrm{C}$ to $23^{\circ} \mathrm{C}$ range, ${ }^{31}$ this yields $\Gamma_{\mathrm{RBCs}}=\Gamma_{\text {water }}\left(1+0.403 f_{150 \mathrm{~g} / \mathrm{L}}\right)$, which shows that the baseline and slope are within the same magnitude as those found for the sulfates.

The approach used to estimate the Grüneisen parameter assumed that it has no dependence on wavelength. Figure 8 shows, for the 740- to 1100 -nm wavelength range (the NIR window), the ratio between the peak-to-peak photoacoustic amplitude and the $\mu_{\mathrm{a}}$ obtained by fitting an exponential decay to the photoacoustic signal. This ratio is proportional to the Grüneisen parameter, and it is clear from Fig. 8 that it does not have a prominent dependence on wavelength.

\section{Use of Sulfate Compounds in Phantom Architectures}

While the principal aim of this paper is to describe the properties of sulfates as phantom chromophores for multiwavelength PAI, this section gives a brief summary of some of the types of phantom architecture into which they might be usefully incorporated. All-liquid phantoms (formed by a liquid background medium 
and by insertions such as capillary tubes filled with further liquid solutions of interest) can easily be devised and created. Examples of such use of sulfate compounds, and associated photoacoustic images, can be found. ${ }^{45,46}$ The advantages of these phantoms are flexibility, ease of fabrication, ease of testing different properties (by changing media or solutions), and quite crucially, the fact that the physical, chemical, and mechanical properties remain well-known since water can be used as the solvent and Intralipid (+Tween) used as the main scatterer agent. Such constructs can be especially useful in a laboratory setting, where phantom studies are necessary for proof-ofconcept, validation, benchmarking, redesign, and mitigation of uncertainty of multiwavelength and quantitative imaging techniques or systems. For some applications though, the fact that these phantoms are largely fluid can be a disadvantage. Creation of 3-D printed frames has recently been suggested for better control on capillary-based phantom geometries. ${ }^{47}$ An alternative is to use fluid-filled solid phantoms, e.g., those with a solid background where hollow structures such as channels are incorporated. A variety of matrix materials can be used, but materials such as agar or gelatin will require that the hollow spaces to be filled with aqueous solutions are encapsulated in tubes (walled channels). ${ }^{16}$ Hydrophobic and more long-lasting materials such as $\mathrm{PVCP}^{8}$ or $\mathrm{PDMS}^{48}$ can instead be used to circumvent the need for tubes, forming wall-less channels that can be refilled and used multiple times. An example of such PVCP constructs and obtained images (though with chloride solutions) can be found in Ref. 14. For cases where long-term stability and architectural versatility are key, such as in quality assurance and standardization of commercial or medical-use multiwavelength PA equipments, fully solid phantoms (solid matrix and inclusions) may need to be considered. In this case, the role of sulfates - or in fact any other additives — needs to be considered with great care. As found for other newly devised additive + matrix composites, ${ }^{10,13,21}$ it may be that the optical, thermoelastic, and mechanical behavior of sulfate compounds in the matrix may differ from those found when dissolved solely in water and Intralipid. Further assessment of suitability and characterization on a matrix-by-matrix case is required.

\section{Summary}

The data shown in this paper support the conclusion that the chromophores $\mathrm{CuSO}_{4}$ and $\mathrm{NiSO}_{4}$ display optical and photoacoustic characteristics that make them especially well suited for use in multiwavelength PAI phantom studies. Their optical behavior is linear and predictable-absorption of each species varies linearly with concentration without alterations of spectral shape, and mixtures of the two species yield an absorption that can be explained as a linear combination of their individual absorption contributions. The compounds have interesting and unique spectra, and the concentrations needed to yield absorption values within a physiological range are below the solubility limit. The sulfates displayed long-term photostability (no indication of photobleaching), as well as resistance to transient photobleaching (no saturable absorption phenomena or groundstate depletion), and are therefore suitable for exposure to the high peak power laser pulses typically used in PAI, even when exposed to the high number of pulses required when using some PAI systems. In addition, solutions with tissue-realistic scattering can be prepared by mixing sulfates and Intralipid, as long as an appropriate emulsifier (here Tween-20) is used before mixing with the Intralipid. Finally, the Grüneisen parameter of aqueous solutions of $\mathrm{NiSO}_{4}$ and $\mathrm{CuSO}_{4}$ was found to be larger than that of water, to increase linearly with concentration and to be comparable to that of blood. Though the focus of this study was assessing the suitability of aqueous solutions of sulfate salts as stable and versatile chromophores for phantom studies, adopting encapsulation techniques (e.g., incorporation in liposomes ${ }^{49}$ ) may further render them attractive as in vivo contrast agents.

\section{Disclosures}

The authors have no relevant financial interests in this article and no potential conflicts of interest to disclose.

\section{Acknowledgments}

This work was supported by an iCASE studentship jointly funded by the UK's Engineering and Physical Sciences Research Council and National Physical Laboratory. The authors would like to thank Peter Brecht for suggesting sulfates salts as potential suitable candidates for study, Ollie Ogunlade for suggesting exploring Tween, and Jan Laufer and Jens Buchmann for useful discussions.

\section{References}

1. P. Beard, "Biomedical photoacoustic imaging," Interface Focus 1(4), 602-631 (2011)

2. J. Yao and L. V. Wang, "Photoacoustic tomography: fundamentals, advances and prospects," Contrast Media Mol. Imaging 6(5), 332-345 (2011).

3. J. Brunker et al., "Photoacoustic imaging using genetically encoded reporters: a review," J. Biomed. Opt. 22(7), 070901 (2017).

4. V. Gujrati, A. Mishra, and V. Ntziachristos, "Molecular imaging probes for multi-spectral optoacoustic tomography," Chem. Commun. 53(34), 4653-4672 (2017).

5. J. Weber, P. C. Beard, and S. E. Bohndiek, "Contrast agents for molecular photoacoustic imaging," Nat. Methods 13(8), 639-650 (2016).

6. B. Cox et al., "Quantitative spectroscopic photoacoustic imaging: a review," J. Biomed. Opt. 17(6), 061202 (2012).

7. F. M. Brochu et al., "Towards quantitative evaluation of tissue absorption coefficients using light fluence correction in optoacoustic tomography," IEEE Trans. Med. Imaging 36(1), 322-331 (2017).

8. G. M. Spirou et al., "Optical and acoustic properties at $1064 \mathrm{~nm}$ of polyvinyl chloride-plastisol for use as a tissue phantom in biomedical optoacoustics," Phys. Med. Biol. 50(14), N141-N153 (2005).

9. C. Avigo et al., "Organosilicon phantom for photoacoustic imaging," J. Biomed. Opt. 20(4), 046008 (2015).

10. J. R. Cook, R. R. Bouchard, and S. Y. Emelianov, "Tissue-mimicking phantoms for photoacoustic and ultrasonic imaging," Biomed. Opt. Express 2(11), 3193-3206 (2011).

11. S. E. Bohndiek et al., "Development and application of stable phantoms for the evaluation of photoacoustic imaging instruments," PLoS One 8(9), e75533 (2013).

12. W. C. Vogt et al., "Biologically relevant photoacoustic imaging phantoms with tunable optical and acoustic properties," J. Biomed. Opt. 21, 101405 (2016).

13. J. Laufer, E. Zhang, and P. Beard, "Evaluation of absorbing chromophores used in tissue phantoms for quantitative photoacoustic spectroscopy and imaging," IEEE J. Sel. Top. Quantum Electron. 16(3), 600-607 (2010).

14. M. Fonseca et al., "Characterisation of a phantom for multiwavelength quantitative photoacoustic imaging," Phys. Med. Biol. 61, 4950-4973 (2016).

15. M. O. Culjat et al., "A review of tissue substitutes for ultrasound imaging," Ultrasound Med. Biol. 36(6), 861-873 (2010).

16. B. W. Pogue and M. S. Patterson, "Review of tissue simulating phantoms for optical spectroscopy, imaging and dosimetry," J. Biomed. Opt. 11(4), 041102 (2006). 
17. J. Laufer et al., "In vitro characterization of genetically expressed absorbing proteins using photoacoustic spectroscopy," Biomed. Opt. Express 4, 2477 (2013).

18. A. Marcano O, N. Melikechi, and G. Verde, "Shift of the absorption spectrum of organic dyes due to saturation," J. Chem. Phys. 113(14), 5830-5835 (2000).

19. J. R. Rajian et al., "Drug delivery monitoring by photoacoustic tomography with an ICG encapsulated double emulsion," Opt. Express 19, 14335-14347 (2011).

20. A.-K. Kirchherr, A. Briel, and K. Mäder, "Stabilization of indocyanine green by encapsulation within micellar systems," Mol. Pharm. 6(2), 480-491 (2009).

21. W. U. Malik and M. Muzaffar, "Biuret reaction of transfusion gelation. II. Influence of some bivalent metal ions on absorption spectrum of nickel-transfusion gelation complex," Adv. Synth. Catal. 30(3-4), 145-148 (1965).

22. S. L. Jacques, "Optical properties of biological tissues: a review," Phys. Med. Biol. 58(14), 5007-5008 (2013).

23. D.-K. Yao et al., "Photoacoustic measurement of the Grüneisen parameter of tissue," J. Biomed. Opt. 19(1), 017007 (2014).

24. T. Stahl, T. Allen, and P. Beard, "Characterization of the thermalisation efficiency and photostability of photoacoustic contrast agents," Proc. SPIE 8943, 89435H (2014).

25. G. A. Pang et al., "Photoacoustic signal generation in gold nanospheres in aqueous solution: signal generation enhancement and particle diameter effects," J. Phys. Chem. C 120(48), 27646-27656 (2016).

26. Y.-S. Chen et al., "Silica-coated gold nanorods as photoacoustic signal nanoamplifiers," Nano Lett. 11(2), 348-354 (2011).

27. R. Su et al., "Toward functional imaging using the optoacoustic 3D whole-body tomography system," Proc. SPIE 7564, 756423 (2010).

28. H.-P. Brecht et al., "Whole-body three-dimensional optoacoustic tomography system for small animals," J. Biomed. Opt. 14(6), 064007 (2009).

29. K. Wang et al., "Investigation of iterative image reconstruction in threedimensional optoacoustic tomography," Phys. Med. Biol. 57, 53995423 (2012).

30. E. V. Petrova et al., "Temperature dependence of Grüneisen parameter in optically absorbing solutions measured by $2 \mathrm{D}$ optoacoustic imaging," Proc. SPIE 8943, 89430S (2014).

31. W. Haynes, CRC Handbook of Chemistry and Physics, 93rd ed., Taylor \& Francis, Boca Raton, Florida (2012).

32. E. V. Petrova et al., "Some properties of aqueous solutions of $\alpha-\mathrm{NiSO}_{4}$ $6 \mathrm{H}_{2} \mathrm{O}$," Crystallogr. Rep. 57, 579-584 (2012).

33. J. A. Balderas-Lopez and J. Díaz-Reyes, "Photoacoustic technique in the transmission configuration for quantitative analysis of liquids," Rev. Mex. Fis. 57, 452-459 (2011).
34. A. Arnold, E. Bascetta, and F. Gunstone, "Effect of sodium chloride on pro-oxidant activity of copper (ii) in peroxidation of phospholipid liposomes," J. Food Sci. 56(2), 571-573 (1991).

35. "Emulsion breaking techniques for oil in water solvent extractions," Technical Report, Spectro Scientific, Chelmsford, Massachusetts (2015).

36. P. Walstra, Physical Chemistry of Foods, CRC Press, New York (2002).

37. L. Cui et al., "Effects of salts on oxidative stability of lipids in tween-20 stabilized oil-in-water emulsions," Food Chem. 197, 1130-1135 (2016).

38. G. Y. Huang et al., "Effect of surfactants on dispersion property and morphology of nano-sized nickel powders," Trans. Nonferrous Metals Soc. China 24(11), 3739-3746 (2014).

39. S. A. Prahl, M. J. C. van Gemert, and A. J. Welch, "Determining the optical properties of turbid media by using the adding-doubling method," Appl. Opt. 32(4), 559 (1993).

40. H. J. van Staveren et al., "Light scattering in Intralipid-10\% in the wavelength range of 400-1100 nm," Appl. Opt. 30(31), 4507-4514 (1991).

41. E. Petrova et al., "Using optoacoustic imaging for measuring the temperature dependence of Grüneisen parameter in optically absorbing solutions," Opt. Express 21(21), 25077-25090 (2013).

42. C.-T. A. Chen, L.-S. Chen, and F. Millero, "Speed of sound in $\mathrm{NaCl}$, $\mathrm{MgCl}_{2}, \mathrm{Na}_{2} \mathrm{SO}_{4}$, and $\mathrm{MgSO}_{4}$ aqueous solutions as functions of concentration, temperature, and pressure," J. Acoust. Soc. Am. 63, 1795-1800 (1978).

43. H. Azhari, "Appendix A-typical acoustic properties of tissues," in Basics of Biomedical Ultrasound for Engineers, pp. 313-314, John Wiley \& Sons, Inc., Hoboken, New Jersey (2010).

44. E. V. Savateeva et al., "Optical properties of blood at various levels of oxygenation studied by time resolved detection of laser-induced pressure profiles," Proc. SPIE 4618, 63-75 (2002).

45. M. Fonseca et al., "Three-dimensional photoacoustic imaging and inversion for accurate quantification of chromophore distributions," Proc. SPIE 10064, 1006415 (2017).

46. J. Buchmann et al., "Experimental validation of a Monte-Carlo-based inversion scheme for 3D quantitative photoacoustic tomography," Proc. SPIE 10064, 1006416 (2017).

47. S. J. Arconada-Alvarez et al., "The development and characterization of a novel yet simple 3D printed tool to facilitate phantom imaging of photoacoustic contrast agents," Photoacoustics 5, 17-24 (2017).

48. J. Hwang et al., "Polydimethylsiloxane tissue-mimicking phantoms for quantitative optical medical imaging standards," Proc. SPIE 10056, 1005603 (2017).

49. M. Wehbe et al., "Nanoscale reaction vessels designed for synthesis of copper-drug complexes suitable for preclinical development," PLoS One 11(4), e0153416 (2016).

Biographies for the authors are not available. 